management to date. As of March 2021 mortality was only $1 \%$ with just a single patient dying of a suspected dysrhythmia. There is a lack of international guideline on timing of follow up investigation in routine management of channelopathies, but our cohort suggests that routine Holter monitoring in asymptomatic LQTS and BrS does not significantly alter sudden cardiac death (SCD) risk management. We educate our ICC channelopathy patients' to report their symptoms to facilitate prompt rhythm assessment, and given pressures on current health systems, perhaps focusing on these symptomatic patients would be an appropriate use of resources.

\section{OUR LEFT BUNDLE BRANCH PACING EXPERIENCE: A SINGLE CENTRE STUDY}

R Walsh, F Kehoe, S Frohlich, N Murphy, I Lyne. Beacon Hospital, Dublin, Ireland

\subsection{6/heartjnl-2021-ICS.28}

Background Chronic ventricular pacing results in dyssynchronous ventricular activation and may result in pacing-induced cardiomyopathy. Conduction system pacing (CSP) allows for physiologic ventricular activation. Left bundle branch pacing (LBBp) has emerged in recent time as an alternative to Hisbundle pacing. It offers the physiological ventricular activation while avoiding the difficulty in targeting the His-bundle and maintaining stable long term lead parameters. Here we describe our experience LBBp.

Objective To describe the LBBp experience and compare the learning curve associated with LBBp to that described with His-bundle pacing.

Methods This study is a retrospective and observational study. Our patient cohort of 90 patients was collected between November 2019 to March 2021. We included all patients who underwent LBBp in this period and examined them at 2 and 6 months post operatively. We further divided these patients into four quartiles to further examine our learning curve. They were examined on age, gender, diagnosis, co-morbidities, NYHA score, tricuspid regurgitant velocity, LVEF, LVEDd, procedure time, fluoroscopy time, and complications. We examined pre- and post- QRS, pre- and post- QRS axis, selective LBBp at implant and follow up, and implant pacemaker parameters and at follow-up. Data was collected and analysed.

Results A total of $64.5 \%$ of patients were male, mean age was 76 years. $15.1 \pm 37 \%$ were LBBB with $\mathrm{HF}, 51.1 \pm \%$ were AVN block/AF with AVN ablation, and 30.2\% were sick sinus syndrome. The majority of patients were NYHA I or II. The mean EF preimplant was $48.3 \%$. Average pre- and immediate post- QRS was $126.7 \mathrm{~ms}$ and $126.4 \mathrm{~ms}$ respectively. At a mean follow up of 5 months QRS average was $119.3 \mathrm{~ms}$. Mean thresholds at follow up were $0.73 \pm 0.35 \mathrm{mV}, 0.72 \pm 0.22 \mathrm{mV}$, and $0.8 \pm 0.27 \mathrm{mV}$ at implant, 2 months, and 6 months respectively. Mean procedure time was $71 \pm 31.9$ minutes, with a mean fluoroscopy time of $11.7 \pm 8.6$ minutes. One patient required lead revision with displacement from the interventricular septum, one patient had a mild mediastinal bleed requiring transfusion, and 3 patients that failed LBBp insertion and were converted to standard techniques. No septal perforation, tricuspid valve injury, AV fistula or other pacemaker complication occurred. No complications were seen during follow-up. Conclusion Left bundle branch pacing offers a potential improvement on His-bundle pacing. Thresholds at implant were considerably lower, remained stable during follow-up, and sensing was improved compared to His-bundle pacing. Procedure time reduced as our experience grew, this varied with type of device inserted, as well as fluoroscopy time. Left bundle branch pacing is accessible with a comparable learning curve to His-bundle pacing. However, further large multicentre studies are needed to evaluated left bundle branch pacing further.

\section{THE ASSOCIATION BETWEEN NOVEL ST2, BNP, ATRIAL FIBRILLATION AND HEART FAILURE}

K Al-Wahaibi, I Khan, M Smith, T Leong. Hermitage Medical Clinic, Dublin, Ireland

\subsection{6/heartjnl-2021-ICS.29}

Background/Introduction ST2, a novel marker of fibrosis has been proposed as a novel biomarker for heart failure. There is paucity of data suggesting its association with Atrial Fibrillation (AF), in particular, with regards to the its independent effect after adjustment for clinical factors and traditional biomarkers such as BNP. There is increasing evidence that inflammation and fibrosis are important players in the pathogenesis of AF, the most common arrhythmia that is associated with considerable morbidity. Purpose We sought to examine the association of ST2 with AF and its possible incremental value in combination with traditional biomarkers already used in routine clinical practice such as BNP.

Methods Unselected patients presenting to a Cardiology service who had BNP for clinical reasons, concomitantly had ST2 sent. These patients included those with heart failure, acute coronary syndromes and AF, as well as patients attending cardioversion and cardiac catheterisation. The association between ST2, BNP and clinical factors was examined. ST2 was examined in tertiles as its distribution was highly skewed. Raised BNP was defined as usual $(\geq 100 \mathrm{pg} / \mathrm{ml})$.

Results Of the 619 patients, the mean age was 69 years and $66 \%$ were male. The prevalence of co-morbidities were: Coronary heart disease - CHD (41\%), Atrial Fibrillation - AF (30\%), Heart Failure - HF (20\%), Chronic Kidney Disease CKD (23\%). The mean eGFR was $68 \mathrm{ml} / \mathrm{h}$ and the mean LV ejection fraction was 55\%. Both ST2 and BNP levels were significantly higher in patients with heart failure, CKD and AF. Mean levels of ST2 were (in $\mathrm{ng} / \mathrm{ml}$ ): HF vs no HF (65 vs. $38, \mathrm{p}=0.0001)$ and in CKD vs. no CKD (49 vs. $41, \mathrm{p}=$ 0.001 ), CAD vs no CAD (43 vs. $42, p=0.3$ ). Mean levels of $\mathrm{BNP}$ were (in $\mathrm{pg} / \mathrm{ml})$ : $\mathrm{HF}$ vs no $\mathrm{HF}$ (579 vs. $131, \mathrm{p}=$ $0.0001)$, CKD vs. no CKD (379 vs. $173, \mathrm{p}=0.0001)$, CAD vs. no CAD (239 vs. $206, p=0.5)$. In patients with $\mathrm{AF}$, levels of both ST2 and BNP were higher, as were their mean ages

\begin{tabular}{|c|c|c|c|}
\hline \multirow[t]{2}{*}{$\begin{array}{l}\text { Abstract } 29 \text { Tabl } \\
\text { and AF }\end{array}$} & \multicolumn{3}{|c|}{ Comparison between patients without $\mathrm{AF}$} \\
\hline & No AF & AF & P value \\
\hline Age in years (SD) & $67(10)$ & $75(9)$ & $<0.0001$ \\
\hline Male sex & $65 \%$ & $69 \%$ & 0.44 \\
\hline \multicolumn{4}{|c|}{ Comorbidities/Risk Factors } \\
\hline Chronic heart failure & $13 \%$ & $36 \%$ & $<0.0001$ \\
\hline Chronic kidney disease & $18 \%$ & $34 \%$ & 0.003 \\
\hline Coronary heart disease & $46 \%$ & $29 \%$ & 0.001 \\
\hline $\operatorname{LVEF}(\mathrm{SD})$ & $65 \%(13 \%)$ & $53 \%(11 \%)$ & 0.005 \\
\hline \multicolumn{4}{|l|}{ Biomarkers } \\
\hline $\mathrm{BNP}(\mathrm{pg} / \mathrm{ml})$ & 158 & 370 & $<0.0001$ \\
\hline $\mathrm{ST} 2(\mathrm{ng} / \mathrm{ml})$ & 40 & 50 & $<0.0001$ \\
\hline hs-Tnl (ng/L) & 454 & 689 & 0.03 \\
\hline Creatinine $(\mu \mathrm{mol} / \mathrm{L})$ & 90 & 108 & $<0.0001$ \\
\hline eGFR $(\mathrm{mL} / \mathrm{min} / 1.73 \mathrm{~m} 2)$ & 67 & 60 & $<0.0001$ \\
\hline
\end{tabular}


Abstract 29 Table 2 Relative risk ratio/odds ratio of Atrial Fibrillation in univariable and multivariable models Model 1: Adjusted for age and sex.

Model 2: Adjusted for model 1 and, LV EF, heart failure, coronary artery disease and eGFR.

Model 3: Adjusted for model 2 and Raised BNP.

Multivariable-adjusted proportional hazards regression models for AF, for ST2 and BNP.

\begin{tabular}{|c|c|c|c|c|c|}
\hline & & Unadjusted & Model 1 & Model 2 & Model 3 \\
\hline \multirow[t]{2}{*}{$\begin{array}{c}\text { ST2 } \\
\text { Tertiles }\end{array}$} & RRR $(95 \% \mathrm{Cl})$ & $\begin{array}{c}1.9 \\
(1.5,2.4)\end{array}$ & $\begin{array}{c}1.6 \\
(1.2,2.0)\end{array}$ & $\begin{array}{c}1.5 \\
(1.1,2.0)\end{array}$ & $\begin{array}{c}1.2 \\
(0.9,1.6)\end{array}$ \\
\hline & P Value & $<0.001$ & $<0.001$ & 0.005 & 0.3 \\
\hline \multirow[t]{2}{*}{$\begin{array}{l}\text { Raised } \\
\text { BNP }\end{array}$} & OR $(95 \% \mathrm{Cl})$ & $\begin{array}{c}10.7 \\
(6.9,17.0)\end{array}$ & $\begin{array}{c}7.8 \\
(4.9,12.4)\end{array}$ & $\begin{array}{c}7.4 \\
(4.3,12.8)\end{array}$ & $\begin{array}{c}7.2 \\
(4.0,13.0)\end{array}$ \\
\hline & P Value & $<0.001$ & $<0.001$ & $<0.001$ & $<0.001$ \\
\hline
\end{tabular}

and prevalence of co-morbidities (see table 1). Both ST2 and BNP correlated with AF (Pearson $r=0.2, p<0.0001$ for ST2 and $\mathrm{r}=0.5, \mathrm{p}<0.0001$ for $\mathrm{BNP})$. There was also a graded association of ST2 with AF $(15 \%, 33 \%$ and $40 \%$ across tertiles of ST2, $\mathrm{p}=0.0001)$. Both ST2 and raised BNP were significant associated with $\mathrm{AF}$ in both univariable and multivariable regression models, and remained independent predictors when adjusted for HF, CHD, LV EF and eGFR (table 2, Model 2 - Relative risk ratio of AF with ST2 across tertiles 1.5, p0.005, and Odds ratio of AF with raised BNP 7.4, $\mathrm{p}<0.001)$. However, ST2 loses significance when BNP is added to the model in combination (table 2 - Model 3).

Conclusions Both ST2 and BNP are independent predictors of AF even after adjustment for heart failure and other clinical factors. When ST2 is used in combination with BNP, raised BNP appears to be a stronger predictor of AF than ST2.

\section{A SURVEY OF KNOWLEDGE AND UNDERSTANDING OF PHARMACOTHERAPY IN STABLE HEART FAILURE AMONGST GENERALISTS}

P Murray, CT O'Connor, V Maher, D Moore. Tallaght University Hospital, Dublin, Ireland

\subsection{6/heartjnl-2021-ICS.30}

Introduction Heart failure (HF) is a commonly encountered clinical entity in the developed world, and is estimated to cost $660 \mathrm{~m}$ euro annually to the Irish exchequer. Evidence supporting the benefit of multi disciplinary care in reduction of readmission rates has prompted the establishment of specialist HF clinics, resulting in improved medication adherence and early recognition and treatment of mild decompensation, thus averting the need for hospital admission. In contrast to this, relatively little emphasis has been placed on the role of the hospital generalist and the general practitioner in the management of stable HF. A small randomised controlled trial comparing multidisciplinary care to routine care in Irish hospitals demonstrated that both models of care reduced the rate of early readmission to nil when patients were clinically stable on target doses of ACE inhibitor prior to discharge. This aim of this survey was to assess knowledge of pharmacotherapeutic options amongst generalists, and identify opportunities for education, in order to further optimise guideline-directed medical therapy while the patient awaits specialist input.
Methods A 14 item questionnaire regarding the indications for HF medications (as per ESC guidelines) was circulated to doctors throughout Ireland working in medicine and general practice; Initially in paper format, with subsequent crossover to web-based format accessed through a link distributed on numerous social media.

Results Total number of respondents was 127, with responses from 27 centres. Practitioners of extraneous specialities $(\mathrm{N}=10)$ were excluded (e.g. surgical/paediatric doctors). $\mathrm{N}=117$ responses analysed. Median number of years of practice was 4 . Table 1 depicts respondents who did not identify heart failure with reduced ejection fraction $(\mathrm{HFrEF})$ as a strong indication for each of the therapies shown, both in absolute numbers and by frequency of grade. When Junior doctors and senior doctors were grouped, there was no statistical difference in knowledge of indication for beta blockers, ACEi and ARNI. There was a higher knowledge base amongst

Abstract 30 Table 1 Numbers of respondents who failed to identify HFrEF as indication for therapy

\begin{tabular}{lllll}
\hline & Beta blocker & ACEi/ARB & MRA & ARNI \\
\hline Total $(\mathrm{N}=117)$ & 30 & 23 & 21 & 30 \\
Intern $(\mathrm{N}=21)$ & 7 & 7 & 6 & 3 \\
SHO $(\mathrm{N}=64)$ & 14 & 11 & 10 & 16 \\
Registrar $(\mathrm{N}=12)$ & 3 & 1 & 2 & 2 \\
SpR $(\mathrm{N}=14)$ & 3 & 2 & 1 & 5 \\
Consultant $(\mathrm{N}=4)$ & 1 & 2 & 1 & 2 \\
GP $(\mathrm{N}=2)$ & 1 & 0 & 0 & 1 \\
\hline
\end{tabular}

Abstract 30 Table 2 Self-reported competency in initiation and up-titration of medications

\begin{tabular}{llllll}
\hline & Beta blocker & ACEi & ARB & MRA & ARNI \\
\hline Total & 89 & 93 & 69 & 52 & 13 \\
Intern & 9 & 10 & 5 & 2 & 0 \\
SHO & 49 & 51 & 37 & 28 & 7 \\
Registrar & 12 & 12 & 10 & 9 & 3 \\
SpR & 14 & 14 & 11 & 12 & 3 \\
Consultant & 4 & 4 & 4 & 1 & 0 \\
GP & 1 & 2 & 2 & 0 & 0 \\
\hline
\end{tabular}

